PROCEEDINGS OF THE

AMERICAN MATHEMATICAL SOCIETY

Volume 127, Number 11, Pages 3181-3186

S 0002-9939(99)05077-7

Article electronically published on July 8, 1999

\title{
THE NECESSARY AND SUFFICIENT CONDITIONS FOR THE GLOBAL STABILITY OF TYPE- $K$ LOTKA-VOLTERRA SYSTEM
}

\author{
TU CAIFENG AND JIANG JIFA
}

(Communicated by Hal L. Smith)

\begin{abstract}
This paper provides necessary and sufficient conditions for the type- $K$ Lotka-Volterra system to have a globally asymptotically stable positive steady state. The generalization of such a result is given.
\end{abstract}

\section{INTRODUCTION}

Consider the Lotka-Volterra system

$$
\dot{x}=\operatorname{diag}(x)(r+M x), \quad x \in R_{+}^{n}, \quad r \in R^{n},
$$

where

$$
M=\left(\begin{array}{cc}
A & -B \\
-C & D
\end{array}\right)
$$

$A$ is a $k \times k$ matrix with nonnegative off-diagonal elements, $D$ is an $(n-k) \times(n-k)$ matrix with the same property and $B \geq 0, C \geq 0$. We call such matrix $M$ a type- $K$ matrix and system $(\mathrm{S})$ a type- $K$ monotone system. There are many papers about the global behavior of monotone systems (S). Some interesting results are due to Takeuchi and Adachi. Let $N=\{1,2, \ldots, n\}$ and let $Q$ be a subset of $N$. In [1], Takeuchi and Adachi proved that if $M$ is stable, then (S) has a unique nonnegative steady state $\bar{x}$ with $\bar{x}_{q}=0$ for $q \in Q$ and $\bar{x}_{r}>0$ for $r \in N \backslash Q$, which attracts all solutions with initial conditions in $\left\{x \in R_{+}^{n}: x_{r}>0\right.$ for $\left.r \in N \backslash Q\right\}$. They also proved that $\bar{x}$ is globally asymptotically stable relative to $\left\{x \in R_{+}^{n}: x_{r}>\right.$ 0 for $r \in N \backslash Q\}$ if and only if

$$
r_{q}+\sum_{j=1}^{n} m_{q_{j}} \bar{x}_{j} \leq 0 \quad \text { for all } q \in Q .
$$

Concerning system (S), as pointed out by Smith [2, p. 872], one of the most interesting problems is whether or not the groups $I=\{1,2, \ldots, k\}$ and $J=\{k+$ $1, \ldots, n\}$ can coexist. The results of Hirsch [3], [4] suggest that the coexistence must take the form of a positive steady state which should be asymptotically stable. Therefore, providing conditions to guarantee that (S) has a globally asymptotically stable steady state is of great interest. Smith [2, Theorem 4.1] gave one set of

Received by the editors January 7, 1998.

1991 Mathematics Subject Classification. Primary 34C11, 92A15.

Key words and phrases. Lotka-Volterra system, steady state, global stability.

This project was supported by the National Natural Science Foundation of China.

(C)1999 American Mathematical Society 
sufficient conditions for the existence of such a steady state which can only be applied to nonobligate systems $(r>0)$. The present authors generalized Smith's result to obligate systems in [5].

The goal of the present paper is to provide the necessary and sufficient conditions for (S) to have a globally asymptotically stable steady state in the positive orthant. Obviously, the essential condition for (S) to have a globally asymptotically stable steady state is that $M$ is stable. Actually, in this paper, we shall consider the more general system

$$
\dot{x}=\operatorname{diag}(x) f(x), \quad x \in R_{+}^{n},
$$

where $D f(x)$ is a type- $K$ matrix as in $(\mathrm{T})$. We call such system $\left(\mathrm{S}^{*}\right)$ a general type- $K$ monotone system. Under the suitable condition

$$
D f(x) \leq_{K} M, \quad \text { for all } \quad x \in R_{+}^{n},
$$

where $M$ is a type- $K$ matrix as in (T) and stable, we shall present necessary and sufficient conditions for $\left(\mathrm{S}^{*}\right)$ to have a globally asymptotically stable steady state. " $\leq_{K}$ " will be defined in the next section. Let $s(M)=\max \operatorname{Re} \lambda$, where $\lambda$ runs through the eigenvalues of $M . M$ is stable, that is, $s(M)<0$ if and only if the principal minors of $M^{+}$alternate in sign as follows:

$$
(-1)^{k}\left|\begin{array}{ccc}
a_{11}^{+} & \ldots & a_{1 k}^{+} \\
\vdots & & \vdots \\
a_{k 1}^{+} & \ldots & a_{k k}^{+}
\end{array}\right|>0, \quad 1 \leq k \leq n,
$$

where $a_{i j}^{+}=\left|a_{i j}\right|$ for $i \neq j$ and $a_{i i}^{+}=a_{i i}$.

\section{The MAIn ReSUlt}

In this section, we will agree on some notation and establish some conventions. Then, the main result will be stated.

Let $R_{+}^{n}=\left\{x \in R^{n}: x_{i} \geq 0,1 \leq i \leq n\right\}$ denote the nonnegative orthant and Int $R_{+}^{n}=\left\{x \in R_{+}^{n}: x_{i}>0,1 \leq i \leq n\right\}$ denote its interior. $x>0$ means $x_{i}>0$ for all $i$.

In this paper, $K$ is a proper cone in $R^{n}$ which is a nonempty closed convex subset of $R^{n}$ with the property $K \cap(-K)=\{0\}$. If the partial order relation is generated by a cone $K$, we write $x \leq_{K} y$ whenever $y-x \in K$. Then $K=R_{+}^{k} \times\left(-R_{+}^{n-k}\right)$ is a cone. Let $x^{1} \in R^{k}, x^{2} \in R^{n-k}$; it is convenient to write $x=\left(x^{1}, x^{2}\right) \in R^{n}$. Then $x \leq_{K} y$, where $x=\left(x^{1}, x^{2}\right), y=\left(y^{1}, y^{2}\right)$ implies $x^{1} \leq y^{1}, x^{2} \geq y^{2}$. For two $n \times n$ type- $K$ matrices $M_{1}$ and $M_{2}, M_{1} \geq_{K} M_{2}$ if and only if $A_{1} \geq A_{2}, B_{1} \geq B_{2}$, $C_{1} \geq C_{2}, D_{1} \geq D_{2}$.

Let $N=\{1,2, \cdots, n\}, I=\{1,2, \cdots, k\}$ and $J=\{k+1, \cdots, n\}$, where $n$ is the dimension of our Euclidean space $R^{n}$. If $L, P$ are nonempty sets of $N$ such that $L \supset I$ and $P \supset J$, then $\bar{L}=N \backslash L$ and $\bar{P}=N \backslash P$ denote their complementary sets in $N . u$ will always represent vectors in $\sharp L$-dimensional Euclidean space $R_{+}^{\sharp L}, v$ in $\sharp \bar{L}$-dimensional Euclidean space $R_{+}^{\sharp \bar{L}}, w$ in $R_{+}^{\sharp P}$ and $z$ in $R_{+}^{\sharp \bar{P}} . \sharp L$ represents the cardinality of $L . \sharp \bar{L}, \sharp P$ and $\sharp \bar{P}$ have the same meanings respectively.

Without loss of generality, we may assume $L=\{1,2, \cdots, k, k+1, \cdots, l\}, k<$ $l<n$, and $P=\{n-p+1, \cdots, k+1, \cdots n\}, p>n-k$. Let $x=(u, v)$ and 
$f(x)=\left(f_{L}(u, v), f_{\bar{L}}(u, v)\right)$. Then we can rewrite the system $\left(\mathrm{S}^{*}\right)$ as

$$
\left\{\begin{array}{l}
\dot{u}=\operatorname{diag}(u) f_{L}(u, v), \\
\dot{v}=\operatorname{diag}(v) f_{\bar{L}}(u, v),
\end{array} \quad u \in R_{+}^{\sharp L}, \quad v \in R_{+}^{\sharp \bar{L}} .\right.
$$

Similarly, let $x=(z, w)$ and $f(x)=\left(f_{\bar{P}}(z, w), f_{P}(z, w)\right)$. Then we can rewrite the system $\left(\mathrm{S}^{*}\right)$ as

$$
\left\{\begin{array}{l}
\dot{z}=\operatorname{diag}(z) f_{\bar{P}}(z, w), \\
\dot{w}=\operatorname{diag}(w) f_{P}(z, w),
\end{array} \quad z \in R_{+}^{\sharp \bar{P}}, \quad w \in R_{+}^{\sharp P} .\right.
$$

Setting $v=0$ and $z=0$ in $\left(\mathrm{S}_{1}\right)$ and $\left(\mathrm{S}_{2}\right)$ respectively, we obtain two subsystems

$$
\dot{u}=\operatorname{diag}(u) f_{L}(u, 0), \quad u \in R_{+}^{\sharp L},
$$

and

$$
\dot{w}=\operatorname{diag}(w) f_{P}(0, w), \quad w \in R_{+}^{\sharp P} .
$$

Because $D f(u, v)$ and $D f(z, w)$ are type- $K$ matrices, $D f_{L}(u, 0)$ is a type- $K_{1}$ submatrix of $D f(u, 0)$ and $D f_{P}(0, w)$ is a type- $K_{2}$ submatrix of $D f(0, w)$, where $K_{1}=R_{+}^{k} \times\left(-R_{+}^{l-k}\right)$ and $K_{2}=R_{+}^{p+k-n} \times\left(-R_{+}^{n-k}\right)$.

We write $\phi_{t}(x)$ for the unique solution $x(t)$ of $\left(\mathrm{S}^{*}\right)$ satisfying $x(0)=x ; \phi_{t}^{L}(u)$, $\phi_{t}^{P}(w)$ are the unique solutions $u(t)$ of $\left(\mathrm{S}_{L}\right)$ and $w(t)$ of $\left(\mathrm{S}_{P}\right)$ respectively, satisfying $u(0)=u$ and $w(0)=w .\left(\phi_{t}^{L}(u)\right)_{I}$ consists of components $\left\{\phi_{t}^{L}(u)\right\}_{i}$ of $\phi_{t}^{L}(u)$ for all $i \in I$, and $\left(\phi_{t}^{P}(w)\right)_{J}$ is defined similarly.

Similar to the result of Takeuchi and Adachi, we have proved the following theorem [7, Theorem 4.1] for the type- $K$ system $\left(\mathrm{S}^{*}\right)$.

Theorem 2.1. Assume that the system $\left(\mathrm{S}^{*}\right)$ is type-K monotone and the condition (C) holds. If there exists a nonnegative steady state $c \in R_{+}^{n}$ with $c_{Q}>0, c_{\bar{Q}}=0$ where we agree on $c=0$ if $Q=\varnothing$, then $c$ attracts all solutions with initial conditions in $\left\{x \in R_{+}^{n}: x_{Q}>0\right\}$ if and only if $f(c) \leq 0$.

Our main result is as follows.

Theorem A. Assume that the condition (C) holds. Then the type-K monotone system $\left(\mathrm{S}^{*}\right)$ has a unique positive steady state which is globally asymptotically stable relative to Int $R_{+}^{n}$ if and only if $\left(\mathrm{S}^{*}\right)$ satisfies the following conditions:

(1) There exists $L \subset N$ with $L \supset I$ such that $\left(\mathrm{S}_{L}\right)$ has a positive steady state $u^{0}$ and $f_{\bar{L}}\left(u^{0}, 0\right)>0$.

(2) There exists $P \subset N$ with $P \supset J$ such that $\left(\mathrm{S}_{P}\right)$ has a positive steady state $w^{0}$ and $f_{\bar{P}}\left(0, w^{0}\right)>0$.

\section{The PRoof of THE MAIN RESUlt}

It is essential to establish some preliminary results before giving the proof of Theorem A.

Theorem 3.1 (Kamke Theorem). Assume that the system $\left(\mathrm{S}^{*}\right)$ is type-K monotone, and $x(t), y(t)$ are the solutions of $\left(\mathrm{S}^{*}\right)$ defined on $a \leq t \leq b$ with $x(a) \leq_{K} y(a)$. Then $x(t) \leq_{K} y(t)$ for all $t \in[a, b]$.

Theorem 3.2. Let the system $\left(\mathrm{S}^{*}\right)$ be a type-K monotone system and let $f(x) \geq_{K}$ 0 for some $x \in R_{+}^{n}$. Then $\left\{\phi_{t}(x)\right\}_{i}$ is nondecreasing if $i \in I$ and $\left\{\phi_{t}(x)\right\}_{j}$ is nonincreasing if $j \in J$ for all $t \geq 0$ for which the solution exists. A similar result holds if $f(x) \leq_{K} 0$. 
Theorem 3.1 is extended in a natural way from cooperative systems to type- $K$ monotone systems (see [2, Theorem 2.4]). Theorem 3.2 is a criterion for the monotonicity of every component of a solution which is generalized from a cooperative system given by Selgrade [6] to a type- $K$ monotone system ([2, p.862]).

The proof of Theorem A (Necessity). Since the condition (C) holds, every solution of the type- $K$ monotone system $\left(\mathrm{S}^{*}\right)$ is bounded by Proposition 4.4 in [7]. Let $p=\left(p_{1}, p_{2}\right)$ be a positive steady state of $\left(\mathrm{S}^{*}\right)$ which is globally asymptotically stable in Int $R_{+}^{n}$. Then the subsystems $\left(\mathrm{S}_{I}\right)$ and $\left(\mathrm{S}_{J}\right)$ have positive steady states $x_{1}^{0}$ and $x_{2}^{0}$, respectively, and $x_{1}^{0} \geq p_{1}, x_{2}^{0} \geq p_{2}$ by Proposition 3.3 in [2].

We claim that $J_{1}=\left\{j \in J: f_{j}\left(x_{1}^{0}, 0\right)>0\right\} \neq \varnothing$. Otherwise, $f_{J}\left(x_{1}^{0}, 0\right) \leq 0$, which implies $f\left(x_{1}^{0}, 0\right) \leq 0$. Then $\left(x_{1}^{0}, 0\right)$ and $p$ are both globally asymptotically stable relative to Int $R_{+}^{n}$ by Theorem 2.1 and the assumption. This is a contradiction and proves our claim.

Set $I_{1}=I \cup J_{1}$. We consider the subsystem

$$
\dot{x}_{I_{1}}=\operatorname{diag}\left(x_{I_{1}}\right) f_{I_{1}}\left(x_{I_{1}}, 0\right) \text {. }
$$

Obviously, $f_{I_{1}}\left(x_{1}^{0}, 0\right) \leq_{K_{1}} 0$ where $K_{1}=R_{+}^{k} \times\left(-R_{+}^{\# J_{1}}\right)$. Then there exists sufficiently small $v \in \operatorname{Int} R_{+}^{\# J_{1}}$ such that $f_{I}\left(x_{1}^{0}, v, 0\right) \leq 0$ because of $\partial f_{I} / \partial x_{J} \leq 0$ and $f_{J_{1}}\left(x_{1}^{0}, v, 0\right)>0$ because of the continuity of $f$. It follows that $\phi_{t}^{I_{1}}\left(x_{1}^{0}, v\right)$ is type- $K_{1}$ nonincreasing from Theorem 3.2. Choose a small $v$ such that $\left(x_{1}^{0}, v\right) \geq_{K_{1}}\left(p_{I}, p_{J_{1}}\right)$. Then $\phi_{t}^{I_{1}}\left(x_{1}^{0}, v\right) \geq K_{1} \phi_{t}^{I_{1}}\left(p_{I_{1}}\right)$ by Theorem 3.1, that is, $x_{1}^{0} \geq\left\{\phi_{t}^{I_{1}}\left(x_{1}^{0}, v\right)\right\}_{I} \geq$ $\left\{\phi_{t}^{I_{1}}\left(p_{I_{1}}\right)\right\}_{I}$ and $v \leq\left\{\phi_{t}^{I_{1}}\left(x_{1}^{0}, v\right)\right\}_{J_{1}} \leq\left\{\phi_{t}^{I_{1}}\left(p_{I_{1}}\right)\right\}_{J_{1}}$ for all $t>0$.

It is easy to prove that $f_{I_{1}}\left(p_{I_{1}}, 0\right) \geq K_{1} 0$. In fact, $f_{I}\left(p_{I_{1}}, 0\right) \geq f_{I}\left(p_{I_{1}}, p_{\bar{I}_{1}}\right)=0$ because of $\partial f_{I} / \partial x_{J} \leq 0$ and $f_{J_{1}}\left(p_{I_{1}}, 0\right) \leq f_{J_{1}}\left(p_{I_{1}}, p_{\bar{I}_{1}}\right)=0$ because of $\partial f_{J_{1}} / \partial x_{\bar{I}_{1}} \geq$ 0 . Then $\phi_{t}^{I_{1}}\left(p_{I_{1}}\right)$ is type- $K_{1}$ nondecreasing by Theorem 3.2. Then $\left\{\phi_{t}^{I_{1}}\left(p_{I_{1}}\right)\right\}_{I} \geq p_{I}$ and $\left\{\phi_{t}^{I_{1}}\left(p_{I_{1}}\right)\right\}_{J_{1}} \leq p_{J_{1}}$ for $t>0$. Thus we have $x_{1}^{0} \geq\left\{\phi_{t}^{I_{1}}\left(x_{1}^{0}, v\right)\right\}_{I} \geq p_{I}$ and $v \leq\left\{\phi_{t}^{I_{1}}\left(x_{1}^{0}, v\right)\right\}_{J_{1}} \leq p_{J_{1}}$. It follows from the type- $K_{1}$ increase of $\phi_{t}^{I_{1}}\left(x_{1}^{0}, v\right)$ that

$$
\lim _{t \rightarrow+\infty} \phi_{t}^{I_{1}}\left(x_{1}^{0}, v\right)=u_{I_{1}} .
$$

Clearly, $u_{I_{1}}>0$, that is, $\left(\mathrm{S}_{I_{1}}\right)$ has a positive steady state $u_{I_{1}}$.

We claim that $f_{\bar{I}_{1}}\left(u_{I_{1}}, 0\right) \leq 0$ is impossible. If not, then $\left(u_{I_{1}}, 0\right)$ is globally asymptotically stable by Theorem 2.1 , contradicting the assumption. Thus, there exists $J_{2}=\left\{j \in \bar{I}_{1}: f_{j}\left(u_{I_{1}}, 0\right)>0\right\} \neq \varnothing$. If $J_{2}=\bar{I}_{1}$, then we choose $L=I_{1}$. Otherwise, setting $I_{2}=I_{1} \cup J_{2}$, we consider the subsystem

$$
\dot{x}_{I_{2}}=\operatorname{diag}\left(x_{I_{2}}\right) f_{I_{2}}\left(x_{I_{2}}, 0\right) \text {. }
$$

Since $f_{j}\left(u_{I_{1}}, 0_{J_{2}}, 0\right)>0$ for $j \in J_{2}$ and $f_{j}\left(u_{I_{1}}, 0_{J_{2}}, 0\right)=0$ for $j \in I_{1}$, we have $f_{I_{2}}\left(u_{I_{1}}, 0_{J_{2}}, 0\right) \leq_{K_{2}} 0$, where $K_{2}=R_{+}^{k} \times\left(-R_{+}^{\#\left(J_{1} \cup J_{2}\right)}\right)$. It is easy to see that $f_{j}\left(p_{I_{1}}, p_{J_{2}}, 0\right) \leq f_{j}\left(p_{I_{1}}, p_{J_{2}}, p_{\bar{I}_{2}}\right)=0$ for $j \in J_{1} \cup J_{2}$ because of $\partial f_{j} / \partial x_{\bar{I}_{2}} \geq 0$ for $j \in$ $J_{1} \cup J_{2}$ and $f_{j}\left(p_{I_{1}}, p_{J_{2}}, 0\right) \geq f_{j}\left(p_{I_{1}}, p_{J_{2}}, p_{\bar{I}_{2}}\right)=0$ for $j \in I$. Then $f_{I_{2}}\left(p_{I_{1}}, p_{J_{2}}, 0\right) \geq_{K_{2}}$ 0 . The same reasoning can conclude that $\left(\mathrm{S}_{I_{2}}\right)$ has a positive steady state $u_{I_{2}}$.

Similarly, $f_{\bar{I}_{2}}\left(u_{I_{2}}, 0\right) \leq 0$ cannot hold. Then there exists $J_{3}=\left\{j \in \overline{I_{2}}\right.$ : $\left.f_{j}\left(u_{I_{2}}, 0\right)>0\right\} \neq \varnothing$. This process must stop at at most the $(n-k-1)$ th-step. We assume the process stops at the $m$ th-step. Let $L=I \cup J_{1} \cup \cdots \cup J_{m}$. Then the subsystem $\left(\mathrm{S}_{L}\right)$ has a positive steady state $u^{0}$ such that $f_{\bar{L}}\left(u^{0}, 0\right)>0$. Clearly, $L \supset I$.

The necessity of (2) can be proved by using the same method. 
(Sufficiency) In fact, the main idea to prove the sufficiency is taken from [5], [7].

First, we prove that if $\left(\mathrm{S}^{*}\right)$ has a positive steady state $\bar{x}$, then $\bar{x}$ is globally asymptotically stable in Int $R_{+}^{n}$.

Since $\bar{x}$ is a positive steady state, for any $x \in R_{+}^{n}$, we have

$$
f(x)-f(\bar{x})=\left[\int_{0}^{1} D f(s x+(1-s) \bar{x}) d s\right](x-\bar{x}) .
$$

The condition $(\mathrm{C})$ shows that

$$
\int_{0}^{1} D f(s x+(1-s) \bar{x}) d s \leq_{K} M
$$

which implies that

$$
\operatorname{diag}(x) f(x) \leq_{K} \operatorname{diag}(x) M(x-\bar{x}) \quad \text { for } \quad x \geq_{K} \bar{x}, \quad x \geq 0,
$$

and

$$
\operatorname{diag}(x) f(x) \geq_{K} \operatorname{diag}(x) M(x-\bar{x}) \quad \text { for } \quad x \leq_{K} \bar{x}, \quad x \geq 0 .
$$

Make a Lotka-Volterra system

$$
\dot{x}=\operatorname{diag}(x)(r+M x), \quad x \in R_{+}^{n} \quad \text { and } \quad r \in R^{n},
$$

where $r=-M \bar{x}$. Obviously, $\bar{x}$ is a positive steady state for (S) and attracts all points in Int $R_{+}^{n}$ by the result of Takeuchi and Adachi [1] mentioned in the introduction. Hence it follows that

$$
\lim _{t \rightarrow+\infty} \phi_{t}(x)=\bar{x} \quad \text { for } \quad x \in \operatorname{Int} R_{+}^{n} \quad \text { with } \quad x \geq_{K} \bar{x} \quad \text { or } \quad x \leq_{K} \bar{x}
$$

from standard differential inequality arguments.

For any $x \in \operatorname{Int} R_{+}^{n}$, there exist $y \geq_{K} \bar{x}$ and $y>0, z \leq_{K} \bar{x}$ and $z>0$ such that $z \leq_{K} x \leq_{K} y$. Applying Theorem 3.1, we have

$$
\phi_{t}(z) \leq_{K} \phi_{t}(x) \leq_{K} \phi_{t}(y) \text { for } t \geq 0 .
$$

Because

$$
\lim _{t \rightarrow+\infty} \phi_{t}(z)=\lim _{t \rightarrow+\infty} \phi_{t}(y)=\bar{x},
$$

we have established that

$$
\lim _{t \rightarrow+\infty} \phi_{t}(x)=\bar{x} \quad \text { for all } \quad x \in \operatorname{Int} R_{+}^{n} .
$$

Since $D f(\bar{x}) \leq_{K} M$, we have $s(D f(\bar{x})) \leq s(M)<0$ from Perron-Frobenius theory (see [2, Thm.2.2]) which implies that $\bar{x}$ is asymptotically stable. Thus $\bar{x}$ is globally asymptotically stable.

Next, we prove that $\left(0, w^{0}\right) \leq_{K}\left(u^{0}, 0\right)$.

Since $u^{0}$ and $w^{0}$ are positive steady states of $\left(\mathrm{S}_{L}\right)$ and $\left(\mathrm{S}_{P}\right)$ respectively, we deduce that

$$
\lim _{t \rightarrow+\infty} \phi_{t}^{L}(u)=u^{0} \quad \text { for } \quad u \in \operatorname{Int} R_{+}^{\# L},
$$

and

$$
\lim _{t \rightarrow+\infty} \phi_{t}^{P}(w)=w^{0} \quad \text { for } \quad w \in \operatorname{Int} R_{+}^{\# P}
$$


from the above result. It is easy to choose $u \in \operatorname{Int} R_{+}^{\# L}$ and $w \in \operatorname{Int} R_{+}^{\# P}$ such that $(0, w) \leq_{K}(u, 0)$. Then

$$
\left(0, \phi_{t}^{P}(w)\right) \leq_{K}\left(\phi_{t}^{L}(u), 0\right) \quad \text { for } \quad t \geq 0
$$

by Theorem 3.1. Therefore, we conclude that

$$
\left(0, w^{0}\right) \leq_{K}\left(u^{0}, 0\right) .
$$

Finally, we prove that $\left(\mathrm{S}^{*}\right)$ has a unique positive steady state which is globally asymptotically stable in $\operatorname{Int} R_{+}^{n}$.

Since $\left(0, w^{0}\right) \leq_{K}\left(u^{0}, 0\right)$, we have $\left(0, w^{0}\right) \leq_{K}\left(z, w^{0}\right) \leq_{K}\left(u^{0}, 0\right)$ for sufficiently small $z>0$. From condition (2), for above $z$, it is easy to obtain that $f\left(z, w^{0}\right) \geq_{K}$ 0 from the continuity and type- $K$ monotonicity of $f$. Then $\phi_{t}\left(z, w^{0}\right)$ is type- $K$ nondecreasing for $t \geq 0$ by Theorem 3.2. We deduce that $\left(0, w^{0}\right) \leq_{K} \phi_{t}\left(z, w^{0}\right) \leq_{K}$ $\left(u^{0}, 0\right)$ for all $t \geq 0$ from Theorem 3.1. Hence, $\phi_{t}\left(z, w^{0}\right)$ is bounded. Consequently, $\phi_{t}\left(z, w^{0}\right)$ converges to some point $\bar{x}$ as $t \longrightarrow+\infty$ and $\left(0, w^{0}\right) \leq_{K} \bar{x} \leq_{K}\left(u^{0}, 0\right)$. It is clear that $\bar{x}_{I} \geq\left\{\left(z, w^{0}\right)\right\}_{I}>0$. Similarly, from the condition (1), for sufficiently small $v$, we have $\phi_{t}\left(u^{0}, v\right) \longrightarrow \tilde{x}$ as $t \longrightarrow+\infty,\left(0, w^{0}\right) \leq_{K} \tilde{x} \leq_{K}\left(u^{0}, 0\right)$ and

$$
\tilde{x}_{J} \geq\left\{\left(u^{0}, v\right)\right\}_{J}>0 .
$$

Choose $z>0, v>0$ to be small enough such that $\left(z, w^{0}\right) \leq_{K}\left(u^{0}, v\right)$. Then $\phi_{t}\left(z, w^{0}\right) \leq_{K} \phi_{t}\left(u^{0}, v\right)$ for $t>0$. So $\bar{x} \leq_{K} \tilde{x}$, namely, $\left(\bar{x}_{I}, \bar{x}_{J}\right) \leq_{K}\left(\tilde{x}_{I}, \tilde{x}_{J}\right)$. This means

$$
\tilde{x}_{I} \geq \bar{x}_{I}>0, \quad \bar{x}_{J} \geq \tilde{x}_{J}>0 .
$$

Hence, we conclude that $\bar{x}>0$ and $\tilde{x}>0$. From the first paragraph of the proof of sufficiency, it follows that $\bar{x}=\tilde{x}$ which is globally asymptotically stable in $\operatorname{Int} R_{+}^{n}$. The proof is completed.

\section{ACKNOWLEDGMENT}

The authors would like to thank the referee for valuable suggestions.

\section{REFERENCES}

1. Y. Takeuchi, and N. Adachi, The existence of globally stable equilibria of ecosystems of the generalized Volterra type, J. Math. Biol. 10 (1980), 401-415. MR 82e:92048

2. H. L. Smith, Competing subcommunities of mutualists and a generalized Kamke Theorem, SIAM J. Appl. Math. 46 (1986), 856-873. MR 87i:92047

3. M. W. Hirsch, Systems of differential equations which are competitive or cooperative I: Limit sets, SIAM J. Math. Anal. 13 (1982), 167-179. MR 83i:58081

4. M. W. Hirsch, Systems of differential equations which are competitive or cooperative, II: Convergence almost everywhere, SIAM J. Math. Anal. 16 (1985), 423-439. MR 87a:58137

5. Tu Caifeng and Jiang Jifa, The coexistence of a community of species with limited competition, J. Math. Anal. Appl. 217 (1998), 233-245. MR 99c:92061

6. J. F. Selgrade, Asymptotic behavior of solutions to single loop positive feedlack systems, J. Differential Equations 38 (1980), 80-103. MR 82a:34038

7. Tu Caifeng and Jiang Jifa, Global stability and permanence for a class of Type $K$ monotone system, submitted to SIAM J. Math. Anal..

Department of Mathematics, The University of Science and Technology of China, Hefei, People's Republic of China

E-mail address: jiangjf@math.ustc.edu.cn 